\title{
Audit of Outcomes of Endoscopic Cholesteatoma Ear Surgery
}

\author{
Ndivhuwo Diale, Estie Meyer, Tashneem Harris \\ Division of Otolaryngology, University of Cape Town, Groote Schuur Hospital, Red Cross War Memorial Hospital, Cape Town, \\ Western Cape, South Africa \\ Email:ndivhuwobele@yahoo.com
}

How to cite this paper: Diale, N., Meyer, E. and Harris, T. (2020) Audit of Outcomes of Endoscopic Cholesteatoma Ear Surgery. International Journal of Otolaryngology and Head \& Neck Surgery, 9, 161-179. https://doi.org/10.4236/ijohns.2020.95020

Received: July 6, 2020

Accepted: August 31, 2020

Published: September 3, 2020

Copyright $\odot 2020$ by author(s) and Scientific Research Publishing Inc. This work is licensed under the Creative Commons Attribution International License (CC BY 4.0).

http://creativecommons.org/licenses/by/4.0/

\section{(c) (i) Open Access}

\begin{abstract}
Background: The objective of cholesteatoma surgery is not only to eradicate disease, but also reduce the risk of recurrence. While the use of the endoscope has been shown to reduce the rate of residual disease, there is currently insufficient data on recidivism and hearing outcomes following exclusive endoscopic use in cholesteatoma ear surgery. Objectives: Auditing outcomes of exclusive endoscopic surgery (EES) for the surgical management of cholesteatoma, with a secondary aim of comparing recidivism and hearing outcomes of 4 different surgical techniques, namely, EES, microscopic canal wall down (CWD), microscopic canal wall up (CWU) and combined endoscopic-microscopic techniques (CEM). Methods: A retrospective chart review was conducted at two tertiary academic hospitals in Cape Town, namely, Red Cross War Memorial Children's Hospital (RCWMH) for patients below13 years and Groote Schuur Hospital (GSH) for patients above 13 years, over a 5 year period, between January 2012 and December 2016. Results: 128 cholesteatoma surgeries overall; 110 patients were from GSH and 18 from RCWMH. Eight RCWMH patients underwent EES, 7 had CWU, 2 had CWD and 1 underwent CEM. Overall recidivism in the RCWMH population was $33 \%(6 / 18)$, 2 underwent EES, 2 underwent a microscopic CWU, 1 had a CWD and 1 underwent CEM. The mean postoperative hearing in this group was $40 \mathrm{~dB}$ from 50.3 decibels $(\mathrm{dB})$ preoperatively. In the GSH group, 23 underwent an EES, 42 had a CWU, 40 underwent CWD and 5 underwent CEM. Overall recidivism for the GSH group was $17 \%$ (19/110). Of those, 7 underwent EES, 8 underwent microscopic CWU, 1 underwent CWD and 3 underwent CEM. Mean postoperative hearing was $47.4 \mathrm{~dB}$ from $48.4 \mathrm{~dB}$ preoperatively. Conclusions: The CWD technique demonstrated superior outcomes. In the GSH group, the EES approach had the same recurrence rate as CWU. Much higher recidivism was observed in the RCWMH group. Management of cholesteatoma requires a highly individualized approach to determine the most appropriate surgical treatment paradigm.
\end{abstract}




\section{Keywords}

Cholesteatoma, Endoscopic, Cholesteatoma Surgery, Recidivism, Outcomes

\section{Introduction}

The objective of cholesteatoma surgery is complete disease resection to keep the ear safe and dry [1].

Before the arrival of endoscopes, the microscope was a widely used surgical tool in cholesteatoma surgery. Many otologic surgeons preferred using it. Certain advantages of the operative microscope still make it invaluable even in the era of the newer endoscopic technique. Amongst other advantages, the microscope is known for its good quality view of the middle ear, mastoid and temporal bone anatomy and pathology; and it affords the surgeon the freedom to use both hands during surgery [2]. Its main limitation is its inability to look around corners, with concerns of possibly missing pathology in the deeper recesses of the middle ear such as the sinus, tympani, facial recess and the attic [3]. As a result, this may necessitate soft tissue retraction and drilling for adequate exposure.

Endoscopic ear surgery has gained acceptance in its role as a complementary tool to the microscope, but perhaps not so much as a technique for exclusive use in cholesteatoma surgery. To improve understanding of this technique, existing literature on endoscopic cholesteatoma ear surgery was thoroughly reviewed.

Operative endoscopy was introduced in ear surgery in the 1990's by Thomassin [4], with the objective of using it as a complementary tool to the microscope. Over the years, this technique has been widely gaining acceptance. Literature defines exclusive endoscopic ear surgery as the sole use of the rigid endoscope to visualize and facilitate middle ear surgery. It is a minimally invasive approach which is indicated for cholesteatoma not extending beyond the confines of the tympanic cavity [4].

There is currently insufficient data on recidivism and hearing outcomes following exclusive endoscopic use in cholesteatoma ear surgery. It has been reported to improve visualization during cholesteatoma surgery. Reports have shown the endoscope to be a superior tool in detecting cholesteatoma hotspots, without the need to remove excessive bone, and avoiding unnecessary need for soft tissue stripping. With EES patient morbidity has been directly reduced by avoiding skin incisions, reduced need for drilling, less postoperative pain and reduced hospital stay. Its cost effectiveness has been found to be comparable to the microscope.

Some pitfalls of EES are well described in literature and includes using one hand to hold the scope, while the other performs surgery, and looking at the screen when operating which could result in an inability to judge the distance 
between the scope and the surgical plane. Concerns regarding safety of the endoscope as a surgical tool have been raised, and this includes possible thermal injury especially when xenon light is used. Fogging of the scope and ear trauma in case of accidental head movement adds to the drawbacks of this technique.

In certain cases, the use of both the microscope and the endoscope is advisable when dealing with cholesteatoma near high-risk middle ear structures such as the facial nerve.

Studies looking at outcomes of cholesteatoma surgery exclusively using the endoscope are few and long-term follow-up was found to be short.

\section{Objectives}

The objective of this study was to audit outcomes of endoscopic ear surgery for the surgical management of cholesteatoma in the group above 13 years of age and the group less than 13 years of age; with a secondary aim of comparing recidivism and hearing outcomes of 4 different surgical techniques for cholesteatoma, namely, exclusive endoscopic (EES), microscopic canal wall down (CWD), microscopic canal wall up (CWU) and the combined endoscopic-microscopic techniques.

\section{Materials and Methods}

\subsection{Definitions}

Cholesteatoma is a growth of abnormal keratinizing squamous epithelium with accumulation of squamous debris in the middle ear cleft and can be either congenital or acquired.

Canal wall up mastoidectomy refers to exenteration of the mastoid air cells with preservation of the posterior external canal wall.

Canal wall down mastoidectomy refers to the exenteration and exteriorization of the mastoid air cells by removal of the posterior external ear canal wall.

Recurrent cholesteatoma is defined as a new cholesteatoma developing from a newly formed, non-self-cleaning retraction pocket.

Residual cholesteatoma is defined as cholesteatoma left behind during initial surgery.

Recidivism refers to both residual and recurrent disease.

Endoscopic ear surgery is defined as the use of the rigid endoscope to perform ear surgery.

Combined endoscopic-microscopic cholesteatoma ear surgery refers to use of both the microscope and the endoscope for eradication of cholesteatoma. The endoscope may be employed either just for visualization to ensure that the disease has been eradicated after resection has been done microscopically, or the endoscope may be used at the start for resection of cholesteatoma confined to the middle ear and attic but then converted to microscopic approach when the disease extends beyond the posterior limit of the lateral semicircular canal. This is the approach adopted for combined procedures at our institutions. 


\subsection{Study Population}

The study was conducted at two tertiary academic hospitals in Cape Town, Red Cross War Memorial Children's Hospital, which is our main referral center for patients under the age of 13 years, and Groote Schuur Hospital, which serves as a major referral hospital for patients above the age of 13 years. For the purpose of this study, the study participants were classified into a category of less than 13 years of age and above 13 years of age.

\subsection{Study Design}

A retrospective chart review was conducted of all patients who underwent primary surgery for cholesteatoma at Red Cross War Memorial Children's Hospital and Groote Schuur Hospital between January 2012 and December 2016. The study's participants, 128 in total, were identified through the theatre booking books over the 5 year period. Follow-up for assessing outcome was initially conducted at 3 months, 6 months and 12 months, followed by 6 monthly visits in the first year following surgery. The study population was divided into four groups, namely populations A, B, C and D. Population A comprised of patients presenting with cholesteatoma who underwent primary exclusive endoscopic cholesteatoma surgery, while population B included those who underwent primary microscopic canal wall up procedure. Population $\mathrm{C}$ had a primary microscopic canal wall down procedure and Population D underwent a primary combined endoscopic-microscopic approach at initial surgery.

The following data was collected and summarized onto a data collection sheet:

- Age

- Sex

- Affected side

- Preoperative otoendoscopic-otomicroscopic findings

- Preoperative CT scan findings: it was a prerequisite for all patients to have preoperative CT scans to aid with decision for the most appropriate surgical method to use

- Primary site of cholesteatoma

- Integrity of ossicular chain

- Degree of pneumatization (i.e. sclerotic, moderately well or well pneumatized)

- Extent of opacification

- Hearing outcomes looking at preoperative and postoperative air conduction $(\mathrm{AC})$, bone conduction $(\mathrm{BC})$, air-bone gaps (ABGs) and pure-tone averages (PTA)

- Site of recidivism

- Disease complications

- Presence of otorrhea

\subsection{Inclusion Criteria}

- All patients who underwent primary surgery endoscopically or microscopi- 
cally or both combined for cholesteatoma between the time period of January 2012 and December 2016.

- High resolution CT scan done pre-operatively.

\subsection{Exclusion Criteria}

- Cholesteatoma revision surgery.

- Patients lost to follow-up were excluded, i.e. those who did not arrive for their 3 months, 6 months and 12 months post-operative reviews.

\subsection{Statistical Analysis}

Data was captured on a customized data collection sheet and entered into Microsoft Excel spreadsheet for analysis. Frequencies and proportions were used to describe categorical data. The Pearson Chi-square test (or Fisher's exact test where cell values were less than 5) was used to test the differences between groups or categories. All analysis was conducted in Stata version 13 (Stata Corp, College Station, Texas). All p-values $<0.05$ were considered statistically significant.

\section{Results}

\subsection{Baseline Characteristics of Study Participants}

Table 1 shows a total of 128 patients that were included in the study, of which 110 were from Groote Schuur Hospital, and therefore, $>13$ years of age, and 18 that were from Red Cross Children's Hospital, and $<13$ years of age (age range for this group was 4 to 13 years). Of the 128 ears, 31 (24\%) underwent an exclusive endoscopic surgical technique, $49(38 \%)$ had a microscopic canal wall up procedure, $42(33 \%)$ underwent a canal wall down procedure, with $6(4 \%)$ having undergone a combined endoscopic-microscopic procedure. Twenty-three of 31 (74\%) patients, >13-year-old group had an exclusive endoscopic procedure, 42 of $49(86 \%)$ had a microscopic canal wall up technique, 40 of 42 (95\%) had a canal wall down procedure, and 5 of 6 (83) underwent a combined endoscopic-microscopic procedure. Eight (26\%) patients from the $<13$-year-old group underwent an exclusive endoscopic resection, $7(14 \%)$ had a microscopic canal wall up procedure, $2(5 \%)$ had a canal wall down mastoidectomy and 1 (17\%) underwent a combined endoscopic-microscopic procedure. There were 60 (47\%) males and 68 (53\%) females. There was a slight predominance of involvement of $67(52 \%)$ right sided ears and 61 (48\%) left sided ears. All were unilateral with no bilateral involvement. The mean follow-up period was 29 months, with a range of 23 - 36 months.

\subsection{Site of Cholesteatoma}

When findings of the $>13$-year-olds and $<13$-year-olds were combined, the commonest site of cholesteatoma was the attic (66 of 128 , which is $51.6 \%$ ), followed by extension into mastoid ( 54 of 128 , which is $43.2 \%$ ), subsequently fol- 
lowed by extension into the sinus tympani (33 of 128, which is $25.8 \%$ ). Extension of disease into the facial recess and Prussak's space had similar proportions $(14.8 \%)$ and extension of the disease into the hypotympanum was the least common ( 4 of 128, which is $3.1 \%$ ). These findings are illustrated in Figure 1 and depicted in Table 2.

Findings in Figure 2 and Table 3 show that in the age group above 13 years, the attic was the commonly involved primary site (58\%) followed by extension of disease into mastoid in $47 \%$, followed by disease extension into sinus tympani in $25 \%$ of cases. Prussak's space was the fourth most frequently involved subsite ( $18 \%$ of cases) followed by extension into the facial recess (15\%). Extension of disease into the hypotympanum was the least common (4\%).

Table 1. Baseline characteristics of the all study participants.

\begin{tabular}{|c|c|c|c|c|c|}
\hline Characteristic & Endoscopic & CWU & CWD & Combined & Total \\
\hline Total ears & $31(24.2 \%)$ & $49(38.3 \%)$ & $42(32.8 \%)$ & $6(4.7 \%)$ & 128 \\
\hline \multicolumn{6}{|c|}{ Sex } \\
\hline Male & $15(48.4 \%)$ & $19(38.8 \%)$ & $21(50.0 \%)$ & $5(83.3 \%)$ & $60(46.9 \%)$ \\
\hline Female & $16(51.6 \%)$ & $30(61.2 \%)$ & $21(50.0 \%)$ & $1(16.7 \%)$ & $68(53.1 \%)$ \\
\hline \multicolumn{6}{|c|}{ Side of ear } \\
\hline Right & $15(48.4 \%)$ & $31(63.3 \%)$ & $19(45.2 \%)$ & $2(33.3 \%)$ & $67(52.3 \%)$ \\
\hline Left & $16(51.6 \%)$ & $18(36.7 \%)$ & $23(54.8 \%)$ & $4(66.7 \%)$ & $61(47.7 \%)$ \\
\hline \multicolumn{6}{|c|}{ Unilateral/bilateral } \\
\hline Unilateral & $31(100.0 \%)$ & $49(100.0 \%)$ & $42(100.0 \%)$ & $6(100.0 \%)$ & $128(100.0 \%)$ \\
\hline Bilateral & $0(0 \%)$ & $0(0 \%)$ & $0(0 \%)$ & $0(0 \%)$ & $0(0 \%)$ \\
\hline \multicolumn{6}{|c|}{ Numbers per surgical technique } \\
\hline$>13$ age group & $23(74.2 \%)$ & $42(85.7 \%)$ & $40(95.2 \%)$ & $5(83.3 \%)$ & $110(85.9 \%)$ \\
\hline$<13$ age group & $8(25.8 \%)$ & $7(14.3 \%)$ & $2(4.8 \%)$ & $1(16.7 \%)$ & $18(14.1 \%)$ \\
\hline
\end{tabular}

Table 2. Site of cholesteatoma in combined $>13$ and $<13$ age group participants.

\begin{tabular}{cccccc}
\hline Site & Endoscopic & CWU & CWD & Combined & Total \\
\hline Attic & $25(80.6 \%)$ & $22(44.9 \%)$ & $14(33.3 \%)$ & $5(83.3 \%)$ & $66(51.6 \%)$ \\
Extension into sinus tympani & $12(38.7 \%)$ & $13(26.5 \%)$ & $7(16.7 \%)$ & $1(16.7 \%)$ & $33(25.8 \%)$ \\
Extension into facial recess & $7(22.6 \%)$ & $7(14.3 \%)$ & $4(9.5 \%)$ & $1(16.7 \%)$ & $19(14.8 \%)$ \\
Extension into Prussak's space & $4(12.9 \%)$ & $14(28.6 \%)$ & $1(2.4 \%)$ & $0(0.0 \%)$ & $19(14.8 \%)$ \\
Extension into hypotympanum & $0(0.0 \%)$ & $2(4.1 \%)$ & $2(4.8 \%)$ & $0(0.0 \%)$ & $4(3.1 \%)$ \\
Extension into mastoid & $5(16.1 \%)$ & $16(32.7 \%)$ & $27(64.3 \%)$ & $6(100.0 \%)$ & $54(42.2 \%)$
\end{tabular}




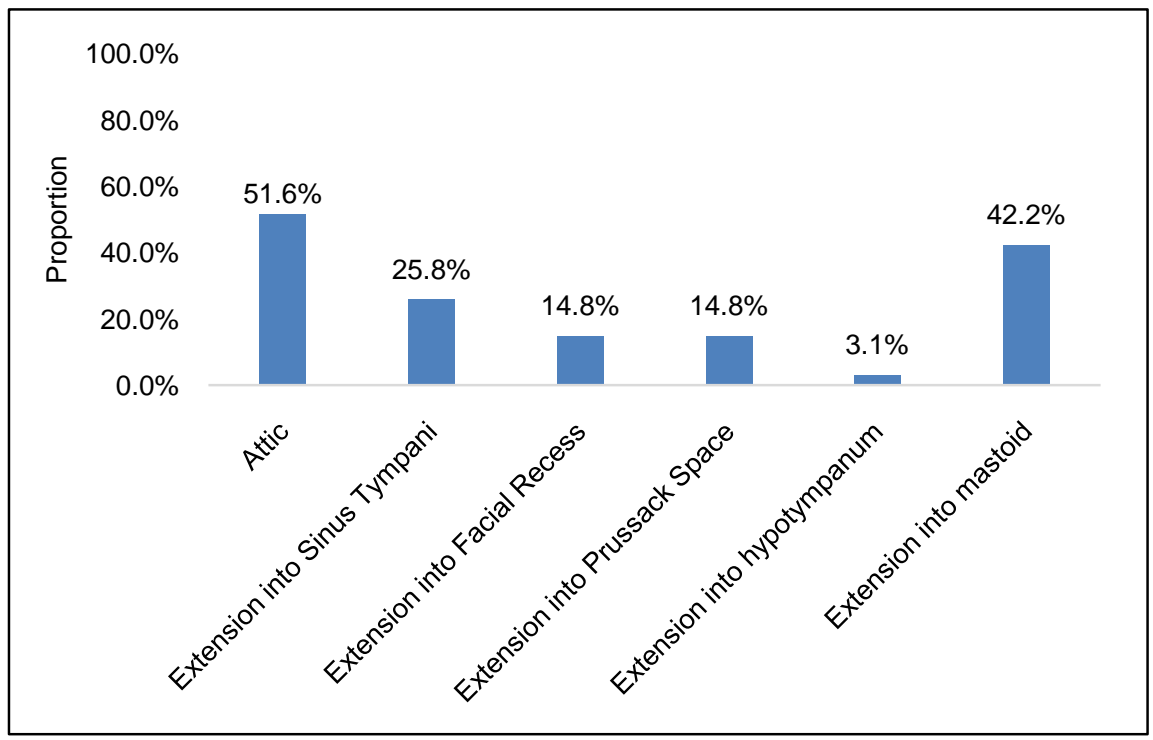

Figure 1. Site of cholesteatoma in both $>13$ and $<13$ age group participants.

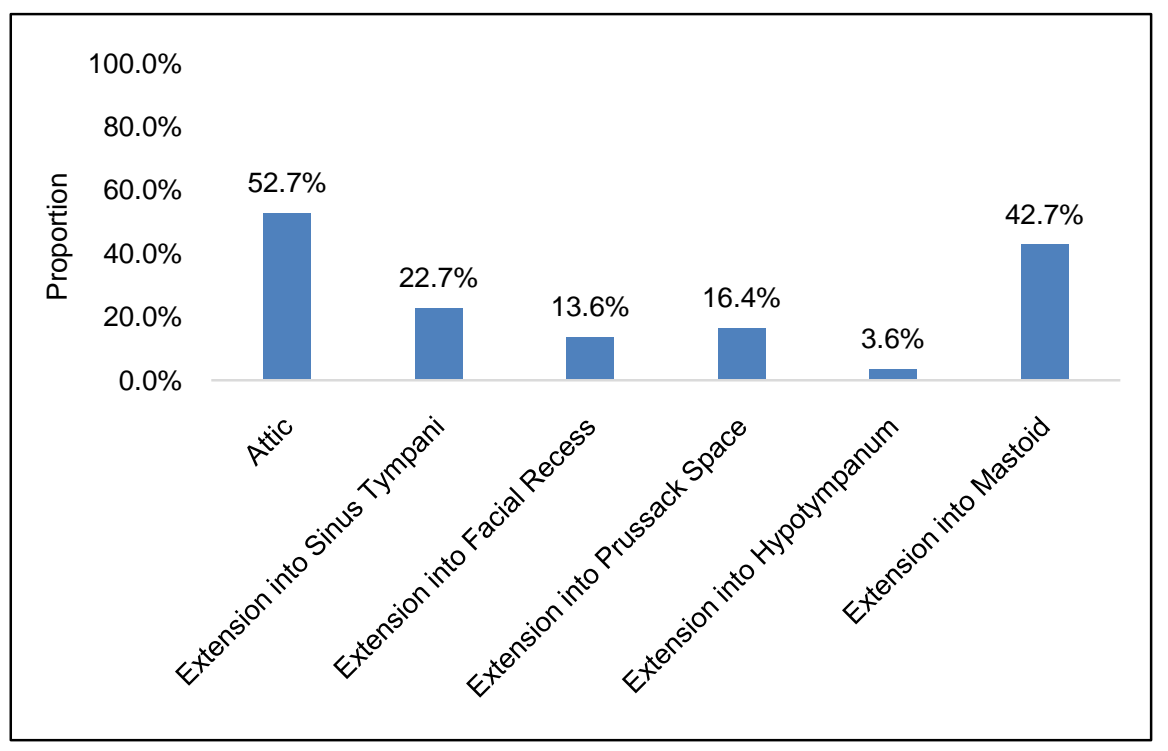

Figure 2. Site of cholesteatoma in the participants $>13$ years old.

Table 3. Site of Cholesteatoma in $>13$ year age group participants.

\begin{tabular}{cccccc}
\hline Site & Endoscopic & CWU & CWD & Combined & Total \\
\hline Attic & $18(78.3 \%)$ & $22(52.4 \%)$ & $14(35.0 \%)$ & $4(80.0 \%)$ & $58(52.7 \%)$ \\
Extension into Sinus Tympani & $8(34.8 \%)$ & $9(21.4 \%)$ & $7(17.5 \%)$ & $1(20.0 \%)$ & $25(22.7 \%)$ \\
Extension into Facial Recess & $5(21.7 \%)$ & $6(14.3 \%)$ & $3(7.5 \%)$ & $1(20.0 \%)$ & $15(13.6 \%)$ \\
Extension into Prussak's Space & $4(17.4 \%)$ & $13(31.0 \%)$ & $1(2.5 \%)$ & $0(0.0 \%)$ & $18(16.4 \%)$ \\
Extension into Hypotympanum & $0(0.0 \%)$ & $2(4.8 \%)$ & $2(5.0 \%)$ & $0(0.0 \%)$ & $4(3.6 \%)$ \\
Extension into Mastoid & $3(13.0 \%)$ & $13(31.0 \%)$ & $26(65.0 \%)$ & $5(100.0 \%)$ & $47(42.7 \%)$
\end{tabular}


When looking exclusively at the $<13$-year-old population, the overall distribution was slightly different. Figure 3 and Table 4 illustrates that the attic and sinus tympani were the commonest sites (each $44.4 \%$ ), followed by extension into the mastoid (38.9\%), the facial recess (22.2\%) and lastly Prussak's space (5.6\%). There were no lesions in the hypotympanum. Of note was that $60 \%$ of ears involved $>$ than 2 sites.

\subsection{Outcomes by Surgical Technique}

The overall recurrence rate was $19.5 \%$ (25 of 128 ) when combining both the above and below 13-year-old groups, this is seen in Figure 4 and summarized in Table 5. This recurrence rate differed between the four intervention groups and was found to be statistically significant $(\mathrm{p}=0.001)$. Recurrence was highest after the combined intervention (4 of 6, which is 66.7\%) and lowest after CWD intervention ( 2 of 42 , which is $4.8 \%$ ). Overall, the attic was the commonest site of recidivism across the four different interventions ( 9 of 25 , which is $36 \%$ ), and the hypotympanum was the least affected.

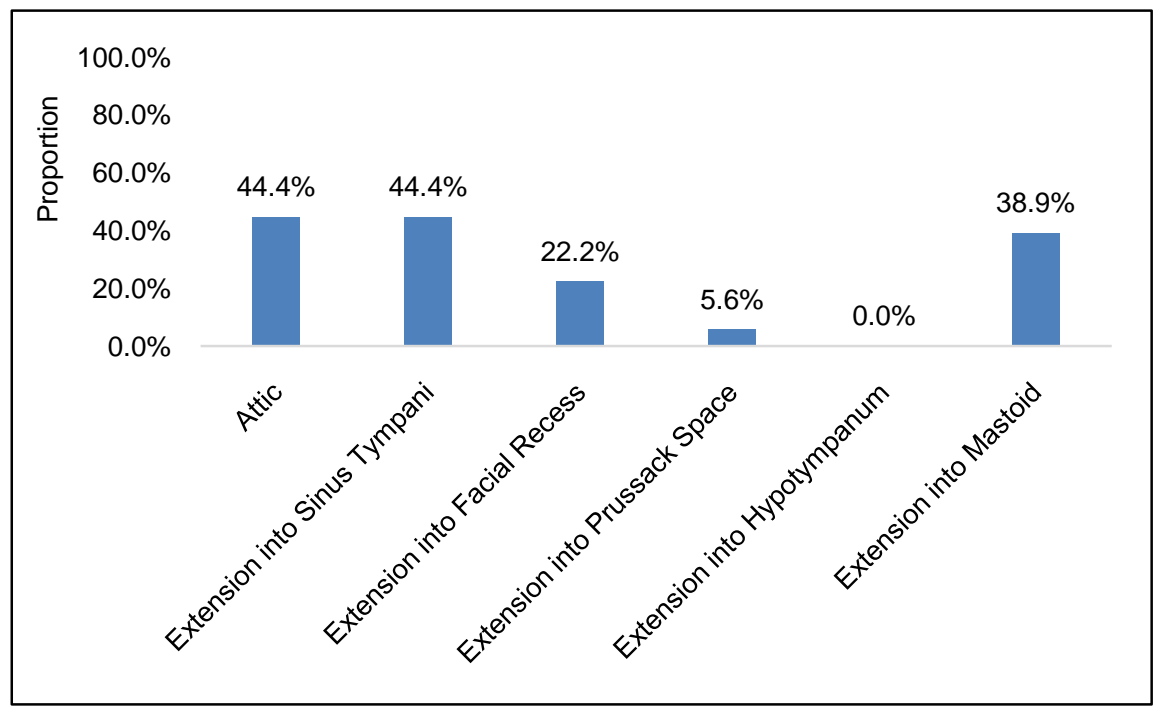

Figure 3. Site of Cholesteatoma in $<13$ age group participants.

Table 4. Site of Cholesteatoma in $<13$ year age group participants.

\begin{tabular}{cccccc}
\hline Site & Endoscopic & CWU & CWD & Combined & Total \\
\hline Attic & $7(87.5 \%)$ & $0(0 \%)$ & $0(0 \%)$ & $1(100.0 \%)$ & $8(44.4 \%)$ \\
Extension into Sinus Tympani & $4(50.0 \%)$ & $4(57.1 \%)$ & $0(0 \%)$ & $0(0 \%)$ & $8(44.4 \%)$ \\
Extension into Facial Recess & $2(25.0 \%)$ & $1(14.3 \%)$ & $1(50.0 \%)$ & $0(0 \%)$ & $4(22.2 \%)$ \\
Extension into Prussak's Space & $0(0 \%)$ & $1(14.3 \%)$ & $0(0 \%)$ & $0(0 \%)$ & $1(5.6 \%)$ \\
Extension into Hypotympanum & $0(0 \%)$ & $0(0 \%)$ & $0(0 \%)$ & $0(0 \%)$ & $0(0 \%)$ \\
Extension into Mastoid & $2(25.0 \%)$ & $3(42.9 \%)$ & $1(50.0 \%)$ & $1(100.0 \%)$ & $7(38.9 \%)$
\end{tabular}




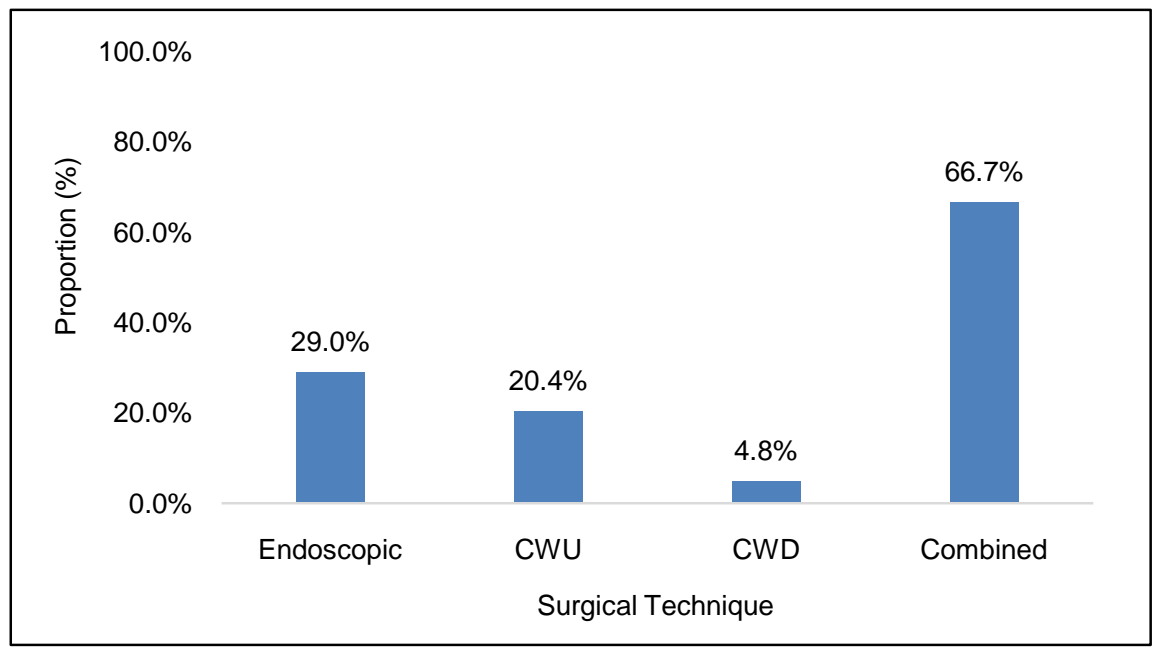

Figure 4. Overall recurrence rate by surgical technique in $>13$ and $<13$ age group participants (overall rate $=19.5 \%$ ).

Table 5. Outcomes by surgical intervention in combined $>13$ and $<13$ age group patients.

\begin{tabular}{cccccc}
\hline Site & Endoscopic & CWU & CWD & Combined & Total \\
\hline Recidivism rate & $9(29.0 \%)$ & $10(20.4 \%)$ & $2(4.8 \%)$ & $4(66.7 \%)$ & $25(19.5 \%)$ \\
& \multicolumn{2}{c}{ Site of recidivism } & & & \\
Attic & $3(33.3 \%)$ & $4(40.0 \%)$ & $1(50.0 \%)$ & $1(25.0 \%)$ & $9(36.0 \%)$ \\
Extension Sinus Tympani & $2(22.2 \%)$ & $2(20.0 \%)$ & $0(0.0 \%)$ & $0(0.0 \%)$ & $4(16.0 \%)$ \\
Extension into Facial Recess & $0(0.0 \%)$ & $3(30.0 \%)$ & $1(50.0 \%)$ & $0(0.0 \%)$ & $4(16.0 \%)$ \\
Extension into Prussak's Space & $1(11.1 \%)$ & $0(0.0 \%)$ & $0(0.0 \%)$ & $0(0.0 \%)$ & $1(4.0 \%)$ \\
Extension into Hypotympanum & $0(0.0 \%)$ & $0(0.0 \%)$ & $0(0.0 \%)$ & $0(0.0 \%)$ & $0(0.0 \%)$ \\
Extension into Mastoid & $3(33.3 \%)$ & $1(10.0 \%)$ & $0(0.0 \%)$ & $3(75.0 \%)$ & $7(28.0 \%)$ \\
\hline
\end{tabular}

Figure 5 clearly demonstrates that in the $>13$-year-old population, the recurrence rate was $17.3 \%$ and the proportion of recurrences by intervention mirrored that of the overall population. This information is summarized in Table 6.

The lowest recidivism rate was seen in the canal wall down group (2.5\%), with unfavourable outcomes noted in the combined endoscopic-microscopic group. A recidivism rate of $19 \%$ was seen in the microscopic CWU and 30\% in the EES group.

The overall recidivism in the $<13$-year-old group is illustrated in Figure 6 and laid out in Table 7. It was higher at 33\%, compared to $17 \%$ for $>13$-year-olds. In this group, recidivism was lowest in the exclusive endoscopic group (25\%), followed by microscopic CWU with almost similar outcomes (28\%). The highest recidivism rate was observed once again in the combined endoscopic-microscopic group (100\%), followed by microscopic CWD recidivism (50\%). The difference was not statistically significant $(\mathrm{p}=0.11)$. In this group however, the numbers were small to make any meaningful comparisons. 


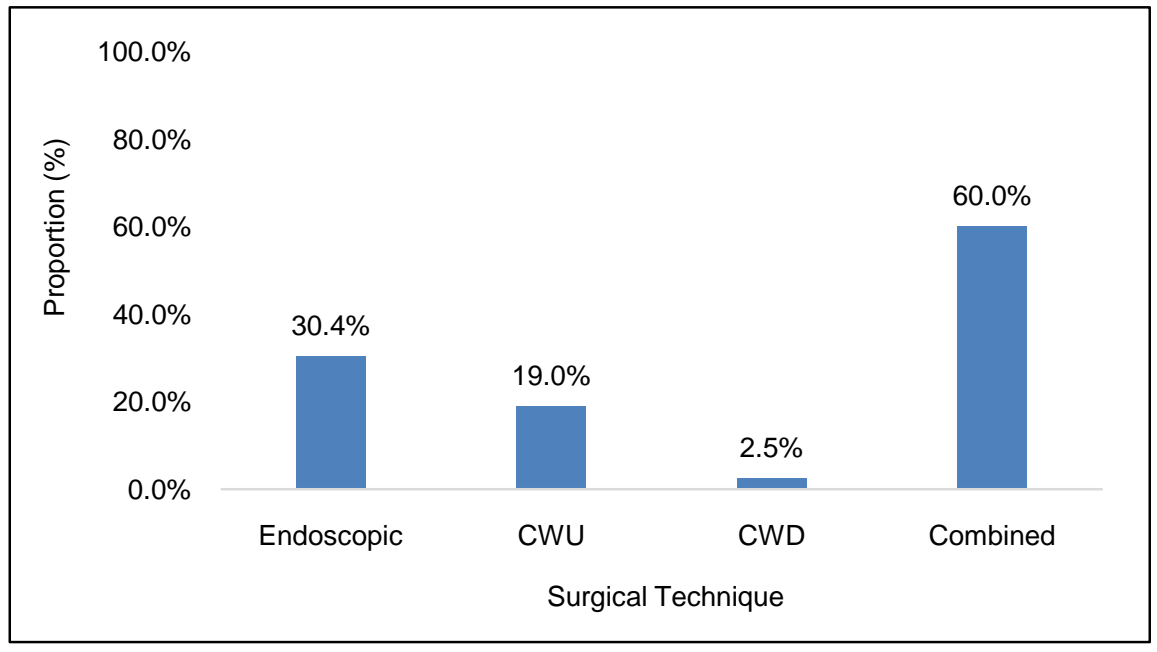

Figure 5. Overall Recurrence rate in $>13$ age group participants by surgical technique.

Table 6. Outcomes by surgical intervention in $>13$ age group participants.

\begin{tabular}{cccccc}
\hline Site & Endoscopic & CWU & CWD & Combined & Total \\
\hline Recurrence & $7(30.4 \%)$ & $8(19.0 \%)$ & $1(2.5 \%)$ & $3(60.0 \%)$ & $19(17.3 \%)$ \\
Site of recurrence & & & \\
Attic & $3(42.9 \%)$ & $3(37.5 \%)$ & $1(100.0 \%)$ & $0(0.0 \%)$ & $7(36.8 \%)$ \\
Extension into Sinus Tympani & $1(14.3 \%)$ & $2(25.0 \%)$ & $0(0.0 \%)$ & $0(0.0 \%)$ & $3(15.8 \%)$ \\
Extension into Facial Recess & $0(0.0 \%)$ & $3(37.5 \%)$ & $0(0.0 \%)$ & $0(0.0 \%)$ & $3(15.8 \%)$ \\
Extension into Prussak's Space & $1(14.3 \%)$ & $0(0.0 \%)$ & $0(0.0 \%)$ & $0(0.0 \%)$ & $1(5.3 \%)$ \\
Extension Hypotympanum & $0(0.0 \%)$ & $0(0.0 \%)$ & $0(0.0 \%)$ & $0(0.0 \%)$ & $0(0.0 \%)$ \\
Mastoid & $2(28.6 \%)$ & $0(0.0 \%)$ & $0(0.0 \%)$ & $3(100.0 \%)$ & $5(26.3 \%)$ \\
\hline
\end{tabular}

\subsection{Site of Recidivism}

The commonest site of recidivism for both groups was the attic and extension of disease into the mastoid, followed by disease extension into sinus tympani and into facial recess. Looking once again at Figure 6 and Table 7, in the $<13$-year-old group, the site of recidivism for microscopic CWU and combined endoscopic-microscopic approaches was confined to the attic, while recidivism in the exclusively endoscopic approach and microscopic CWU approaches extended into the mastoid. In the group $>13$ years, the site of recidivism for the exclusive endoscopic and microscopic CWU approach was confined to the attic, while disease extension into mastoid was seen with exclusive endoscopic and combined endoscopic-microscopic procedures.

\subsection{Hearing Outcomes}

In the $<13$ year age group, the mean postoperative hearing was $40 \mathrm{~dB}$, compared to a preoperative mean of $50.3 \mathrm{~dB}$, see Table 8 . The change was much higher than that of the group $>13$ years of age $(48.4 \mathrm{~dB})$. The overall hearing 


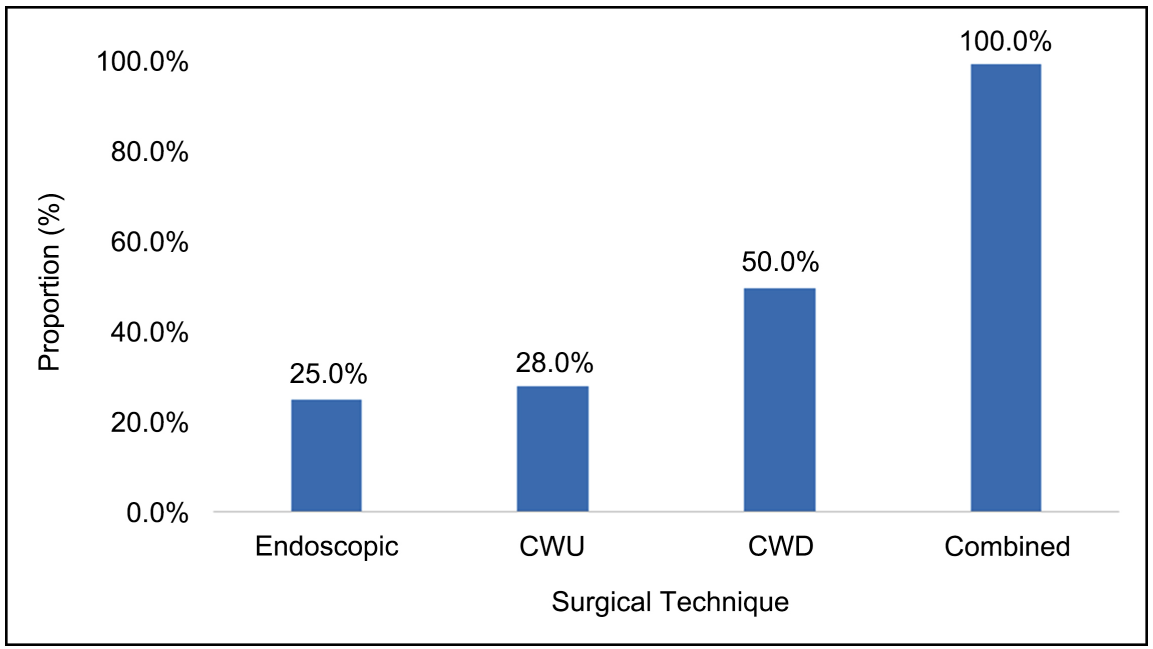

Figure 6. Overall Recurrence rate in the $<13$ age group participants by surgical technique.

Table 7. Outcomes by surgical intervention in $<13$ age group participants.

\begin{tabular}{cccccc}
\hline \multicolumn{1}{c}{ Site } & Endoscopic & CWU & CWD & Combined & Total \\
\hline Recurrence & $2(25.0 \%)$ & $2(28.0 \%)$ & $1(50.0 \%)$ & $1(100.0 \%)$ & $6(33.3 \%)$ \\
Attic & Site of recurrence & & \\
Extension into Sinus Tympani & $1(50.0 \%)$ & $0(0.0 \%)$ & $0(0.0 \%)$ & $0(0.0 \%)$ & $1(16.7 \%)$ \\
Extension into Facial Recess & $0(0.0 \%)$ & $0(0.0 \%)$ & $1(100.0 \%)$ & $0(0.0 \%)$ & $1(16.7 \%)$ \\
Extension into Prussak's Space & $0(0.0 \%)$ & $0(0.0 \%)$ & $0(0.0 \%)$ & $0(0.0 \%)$ & $0(0.0 \%)$ \\
Extension into Hypotympanum & $0(0.0 \%)$ & $0(0.0 \%)$ & $0(0.0 \%)$ & $0(0.0 \%)$ & $0(0.0 \%)$ \\
Extension into mastoid & $1(50.0 \%)$ & $1(50.0 \%)$ & $0(0.0 \%)$ & $0(0.0 \%)$ & $2(33.0 \%)$ \\
\hline
\end{tabular}

Table 8. Pre and post-operative hearing outcomes by intervention group.

\begin{tabular}{|c|c|c|c|c|c|c|}
\hline & Mean scores & Endoscopic & CWU & CWD & Combined & Total \\
\hline \multirow{3}{*}{$\begin{array}{c}>13 \text { age } \\
\text { group }\end{array}$} & Preoperative PTA (dB) & 49.0 & 48.0 & 45.8 & 51.0 & 48.4 \\
\hline & Postoperative PTA (dB) & 42.6 & 43.1 & 41.6 & 54.0 & 47.4 \\
\hline & Delta & 6.4 & 4.9 & 4.2 & -3.0 & 1.0 \\
\hline \multirow{3}{*}{$\begin{array}{c}<13 \text { age } \\
\text { group }\end{array}$} & Preoperative PTA (dB) & 46.3 & 45 & 60 & 50 & 50.3 \\
\hline & Postoperative PTA (dB) & 30.6 & 42 & 42.5 & 45 & 40 \\
\hline & Delta & 15.7 & 3.0 & 17.5 & 5.0 & 10.3 \\
\hline
\end{tabular}

improvement post-operatively in the above 13-year-olds seemed marginal (47.4 $\mathrm{dB}$ post-operatively and $48.4 \mathrm{~dB}$ preoperatively). See Table 8 . Fifty two (41\%) had granulation tissue in the middle ear, while $90 \%$ had ossicular involvement, with the long process of incus being the most frequently eroded ossicle (53\%), followed by stapes superstructure (24\%). 
Analysing ossicular chain findings per technique, the endoscopic group had 2 cases of intact ossicles, 12 cases of eroded long processes of the incus, 5 cases of eroded malleus and 3 cases of eroded stapes superstructures. The CWU group had 6 cases of intact ossicles, 19 eroded long processes of incus, 8 eroded malleus and 11 eroded stapes superstructures. In the CWD group, all ossicles were eroded; 13 involved the long process of incus, 9 involved the malleus and 19 involved the stapes superstructure. Lastly for the combined group, 1 had intact ossicles while 3 had erosion of the long process of incus and 1 had an eroded stapes superstructure. Overall, 47 patients had erosion of long processes of incus, 22 cases had an eroded malleus, 34 had eroded stapes superstructures, while only 9 cases had intact ossicles.

\subsection{Other Interesting Observations}

One hundred patients (79\%) had wet ears at the time of surgery. The chorda tympani nerve was involved by cholesteatoma in $14 \%$ of all cases. Of those, $84 \%$ were sacrificed, with no recidivism observed for the sacrificed nerves regardless of surgical technique. The remaining $16 \%$ of involved chorda tympani had cholesteatoma "peeled off", with 100\% recidivism observed for that group, regardless of surgical technique.

\section{Discussion}

Historically, the management of cholesteatoma was either approached using microscopic canal wall up or microscopic canal wall down techniques. The decision regarding which approach is used is based on a number of factors which includes: the extent of disease, pneumatisation of mastoid, aeration of middle ear and mastoid, reliability of patient to follow-up and surgeon preference. It is well known that a canal wall up technique has a much higher recidivism rate compared to a canal wall down technique.

Recently, endoscopic ear surgeons have been advocating exclusive use of the endoscope in cholesteatoma surgery for disease confined to the middle ear due to lower recidivism rates. In our study, the endoscopic approach for cholesteatoma has comparable results in terms of recidivism with canal wall up mastoidectomy.

We analysed a retrospective study by Ayache [4]. The objectives of the study were to evaluate otoendoscopy as a means of identifying residues of lesions after excision of disease under otomicroscopy in the same stage of surgery and its impact on the frequency of residual cholesteatomas at the time of surgical revision. They had 350 patients. The surgical procedures were divided into closed tympanoplasty via the trans-meatal approach, closed tympanoplasty with antroatticomastoidectomy and open tympanoplasty. Eighty patients (34\%) who presented with an initial location of the disease at the epitympanum underwent complementary exploration by otovideoendoscopy. In 35 cases (44\%), otoendoscopy revealed a residual lesion after an apparently total excision by otomicro- 
scopy during closed tympanoplasty. In 65 cases (76\%), a residual lesion was identified by otoendoscopy during the first stage of surgery in the sinus tympani or on the footplate of the stapes, between the crura of the stapes. Analysis using otoendoscopy reduced the incidence of residual cholesteatomas by identifying lesion extensions that are overlooked under otomicroscopy. In this population, the frequency of CWD was also significantly lower.

A study by Migirov et al. [5] retrospectively looked at recidivism rates in trans-meatal exclusive endoscopic ear surgery (EES). Thirty patients aged 9 to 75 years underwent EES eradication of cholesteatoma between July 2008 and May 2010. The posterior epitympanic space was involved with cholesteatoma in all except one ear. There was no residual disease in 18 patients who were followed-up for $>1$ year, and the diffusion-weighted sequence MRI was negative in 3 patients. Seven additional patients were scheduled to undergo MRI within the next 2 to 3 months. The other 8 patients were satisfied with their clinical results, declined going for a follow-up MRI scan. Their preliminary results indicate that the minimally invasive EES allowed complete eradication of cholesteatoma from the middle ear and its extensions, with minimal morbidity and good functional results. The study showed good results. However the follow-up period of 1 year is very short. Their results however, differ significantly from our EES recidivism of $28 \%$ and $30 \%$ for the $<13$-year-olds and $>13$-year-olds respectively, over a follow-up period of 29 months.

Badr-el-Dine et al. [6] prospectively assessed the value of ear endoscopy in cholesteatoma surgery and whether it improves surgical outcomes. A total of 92 ears were operated on. Eighty two cases were operated on by using canal wall up (CWU) technique, and 10 underwent canal wall down (CWD) procedures. Endoscopically guided ear surgery was incorporated complementary to the microscope. In the primary surgery after completion of microscopic cleaning, the overall incidence of intraoperative residuals detected with the endoscope was $22.8 \%$. Sinus tympani was the most common site of intraoperative residuals in both CWU and CWD groups, followed by the facial recess and the under surface of the scutum in the CWU cases. Three recurrences (8.6\%) were identified with CWU.

Ito et al. conducted a study to confirm that transcanal endoscopic surgery is feasible using rigid endoscopes with an outer diameter of $2.7 \mathrm{~mm}$ and is effective and less invasive for paediatric patients with a narrow ear canal, however, their results don't mention outcomes of their transcanal endoscopic surgery [7].

Marchioni's results [8] showed a higher recidivism, with a recurrence rate of $12.9 \%$ and a residual rate of $19.3 \%$. He had a longer follow up period of 36 months compared to Migirov's study above. Of note, these were all paediatric patients treated with EES approach, between January 2007 and December 2013. Patients presenting with cholesteatoma of the tympanic cavity with no mastoid involvement were included in the first group and underwent EES. Patients with mastoid extension of the pathology were included in the control group and underwent a CWU procedure. Fifty-nine ears of 54 patients were reviewed. Median 
age was 9.6 years (range 4 - 16 years). Thirty-one cholesteatomas underwent an EES approach, while 28 underwent a CWU approach. Recurrence rates were $12.9 \%$ ( 4 ears) for the EES group and $17.2 \%$ ( 5 ears) for the CWU approach. Residual disease was present in 26.6\%: 19.3\% (6 ears) for the EES and 34.4\% (10 ears) for the CWU approach. The mean follow up period was 36 months (range 8 - 88). Kaplan-Meier analysis at 36 months showed a lower recurrence risk for the EES compared with the CWU approach, but this data had no statistical significance $(\mathrm{p}=0.58$ ). This is fairly similar to the findings of our $<13$-year-old group. We had a recidivism of $28 \%$ for CWU and $25 \%$ for EES over a period of 24 to 29 months.

Cohen et al. [9] looked at the rate of residual cholesteatoma during planned second-look procedures in paediatric patients following primary cholesteatoma resection using endoscopic and microscopic operative approaches. Endoscopes were used for inspection in 25 (39\%) primary resections and for dissection in 39 (61\%) primary resections. Extent of disease at the time of primary resection was similar among groups. Cases where the endoscope was used for inspection only or not at all during primary resection had a $24 \%$ rate of residual cholesteatoma at the time of second look compared with a $23 \%$ rate for cases with endoscopic dissection. Rate of mastoidectomy significantly decreased from $63 \%$ to $33 \%$ over the study period, but residual cholesteatoma rates during planned second-look procedures were similar between the study groups.

Tarabichi's study [10] evaluated 8 years of experience with trans-canal endoscopic management of limited attic cholesteatoma. He did a case series on seventy-three ears with limited attic cholesteatoma that underwent endoscopic trans-canal tympanotomy and extended atticotomy to access and completely remove the sac. Their mean follow-up was 43 months. Only five ears required revision for recurrent disease (6.8\%). The recidivism rate was much lower compared to $29 \%$ for our EES overall results . Based on his findings, he concluded that an endoscopic technique allows transcanal, minimally invasive, eradication of limited attic cholesteatoma.

Alicandri-Ciufelli et al. [11] analysed only cholesteatoma treated endoscopically (exclusively or combined) with at least 3 years of follow-up; canal wall down procedures were excluded. The final study group included 244 ears in 234 patients. The mean follow-up was 64.3 months. 166 patients (68\%) free from disease post-operatively, 29 patients (12\%) had recurrence, and 49 patients (20\%) had residual disease. 73 patients (30\%) had a cholesteatoma limited exclusively to the attic, whereas $44(18 \%)$ had also a mesotympanic extension of the disease, 37 (15\%) patients had exclusive involvement of the mesotympanum, 73 (30\%) had antral extension, and 17 (7\%) had mastoid extension. There were 41 patients $(17 \%)$ who were aged $<18$ years, and $203(83 \%)$ were adults. A recurrence or residual was present in 68 adult patients $(33 \%)$, and 10 recurrence or residual in 41 patients aged $<18$ yrs was noted $(24 \%)$. Of 73 patients with cholesteatoma limited exclusively to the attic, 15 experienced residual disease; of 44 
patients who also had a mesotympanic extension, 8 experienced residual pathology at follow-up, and of 37 patients with exclusive mesotympanic involvement, 5 experienced residual disease. Of 73 patients with antral extension, 18 experienced residual pathology, and of 17 patients with mastoid extension, 3 experienced residual pathology. In terms of site of cholesteatoma, their paediatric findings concur with our findings of the attic being the commonest primary site (51\%) and commonest site of recurrence (33\%). Their comment was that the endoscopic results were similar to those reported for classic microscopic techniques in terms of recurrences or residual pathology at long-term follow-up.

Presutti et al. [12] assessed the result of using an endoscope in cholesteatoma surgery and demonstrated how it reduced the incidence of residual disease prospectively. A total of 53 ears with a primarily acquired cholesteatoma were resected. Twenty were resected using a canal wall up (CWU) technique, 6 using a canal wall down (CWD) technique, and in 27 cases, a trans-canal atticotomy was performed. All of the patients underwent explorative and operative endoscopic ear surgery complementary to the operating microscope to uncover and remove residual cholesteatoma. In the primary surgery after completion of microscopic cleaning, the overall incidence of intraoperative residual disease detected with the endoscope was $37.5 \%$, with the sinus tympani being the most common site of intraoperative residuals, followed by the anterior epitympanic recess and protympanum. Out of the 20 CWU cases, 12 second-look endoscopies were performed. Two recurrences were identified, both in the sinus tympani.

Marchioni et al. [13] reported results of 146 ears with a mean follow-up of 31.2 months. Of the 146 patients, $4(2.7 \%)$ patients were diagnosed with recurrence, and 7 (4.8\%) patients had residual disease. Of 33 patients with cholesteatoma limited exclusively to the attic, none experienced residual disease. Fifty-five patients had mesotympanic extension, 2 experienced residual pathologic condition at follow-up. Of 31 patients with antral extension, 2 experienced residual disease, and of 23 patients with mastoid extension, 3 experienced residual disease. When one looks closely at his results for residual disease it would appear that the lowest rate of residual disease is when the cholesteatoma is confined to the attic. As soon the disease is more extensive the risk of leaving behind residual disease appears to be higher. When looking at the sites of involvement in our study, more than $60 \%$ of patients in the overall had more than 2 sites involved by cholesteatoma.

Prasad et al. [14] did a retrospective study to evaluate the outcomes of the modified Bondy's technique performed at their centre and for limited epitympanic cholesteatomas and to debate the benefits of endoscopic surgery for the same indication. Two hundred and sixty nine ears in 258 patients with a minimum of 5-year follow-up that were operated for limited epitympanic cholesteatoma using the modified Bondy's technique were included in the study. There were no recurrent cholesteatomas in their series. Their conclusion was that the 
modified Bondy's technique, which provided excellent postoperative outcomes, is the surgery of choice for limited epitympanic cholesteatomas. They also concluded that the endoscope, despite its better visualization of hidden areas did not provide a distinct overall technical advantage or better results than the microscope.

The important question is: which patients are suitable for exclusive endoscopic technique? Following a literature search, there was no clear patient selection criteria proposed. Both Tarabichi et al. [15] and Glikson et al. [16] recommended endoscopic surgery when the cholesteatoma did not extend posterior to the anterior limb of the lateral semi-circular canal. These are the same criteria we use in both our centres.

When reviewing our CT scans, we paid attention to pneumatisation and ventilation of the temporal bones, as we suspect this may affect recurrence rates. Studies have shown higher incidences of squamous disease with sclerotic mastoids and poor tubal function [17].

Other factors that impact decision making for EES such as patient reliability and preoperative CT scan as a requirement to assess whether disease is confined to middle ear space or extensive, are not adequately addressed in literature.

Comparing recidivism rates between the $>13$-year-old group and $<13$-yearold group, findings in our study show that recidivism rates are higher in the $<13$-year-old population, which concurs with findings in the literature, which shows that children have more aggressive disease caused by continuing eustachian tube dysfunction and hence formation of new retraction pockets [18]. The peaking of recidivism in this group was eleven years. Our study also shows that the exclusive endoscopic approach in paediatrics has a comparable recidivism rate to the microscopic canal wall up procedure. One limitation of our study was that our $<13$-year-old patient numbers were relatively small $(\mathrm{n}=18)$. Larger paediatric studies are obviously needed to derive a meaningful conclusion. Previous studies quoted above looking at cholesteatoma surgery in children reported very favourable results for limited disease.

The reported advantages of endoscopy in cholesteatoma surgery is improved visualization of the hidden sites, and this may have an advantage in reducing residual disease especially in those hidden sites, which include the sinus tympani, facial recess and the attic. In our study, the microscopic canal wall down approach remained the most effective single stage procedure with the lowest recidivism, which was statistically significant $(\mathrm{p}=0.001)$.

Our research study also shows evidence that the combined microscopicendoscopic approach is the least favourable option when disease extends beyond confines of the tympanic cavity. Nogueira et al. [18] looked at outcomes of combined endoscopic-microscopic approach to cholesteatoma in 112 patients. Their recidivism rate was $6.8 \%$, which is a figure almost equivalent to quoted CWD surgery outcomes. Their study does not however, specify whether the canal wall was lowered or not. 
There has been a paradigm shift in the surgical approach to cholesteatoma with intent to restore the tympanomastoid anatomy and physiology. The endoscope no doubt allows a more conservative approach by providing better visualisation of hidden sites thereby enabling preservation of the canal wall. The aim of cholesteatoma surgery however is not only to eradicate disease but also reduce the risk of recurrence. While the use of the endoscope has been shown to reduce the rate of residual disease more studies are required which have a longer follow-up period to assess the risk of recurrent disease.

\section{Conclusions}

The key findings of our study were that the CWD technique demonstrated superior outcomes in both adults and children $<13$ years. In the above 13-year-old group, the EES approach had the same recurrence rate as CWU. While paediatric cholesteatomas have much higher recidivism rates compared to adults, our below 13-year-old group was too small to conclude any statistical difference between the different approaches and further studies are required in this age group.

Based on our data, the choice of appropriate surgical technique should depend on a balance between extent of disease, anatomical favourability, radiological factors and patient reliability. We recommend pre-operative CT scan to assess extent of disease to aid in decision making regarding the most appropriate surgical method to use. To reduce risk factors for recidivism, we advise having a low threshold for taking down the canal wall when disease extends into the mastoid. Although the surgeon's personal preference and experience should also be taken into consideration, an individualized approach must be adopted for all patients.

\section{Limitations}

- In the group below 13 years of age, we had a limited number of patients, statistically, it was not possible to make meaningful conclusions.

- Grouping of participants according to age above or below 13 was due to our two referral hospitals, Red Cross and Groote Schuur, using 13 years as a cut-off age for referring and managing patients. Below 13-year-old are seen at Red Cross only, while those above 13 are referred and managed only in Groote Schuur Hospital.

- Disease extension differed across all 4 study groups, meaning cholesteatomas were not confined to the same sites, this was noted as a potential limitation of the study.

\section{Suggestions for Further Research}

Studies with a long-term follow up and larger cohort studies are necessary to definitively validate the outcomes of the different techniques in cholesteatoma surgery, for a more meaningful conclusion. Studies looking at correct patient se- 
lection for the different surgical method would add quality and standardized approaches. None of the papers considered in this study were randomized trials, so there is always room for bias.

\section{Acknowledgements}

I am deeply grateful to God Almighty for my family who gave constant encouragement and support during this research study. I would like to thank Dr. Tashneem Harris, for her invaluable guidance and supervision of this Masters research study, from the conception of the project to the development of the final manuscript; Dr. Estie Meyer for providing access to her endoscopic ear surgery database as well as contributing to the final manuscript; Dr. Simba Takuva, for his input on statistical analysis; and to the management at Groote Schuur and Red Cross War Memorial Children's Hospitals, for allowing me the opportunity to conduct this research.

\section{Conflicts of Interest}

The authors declare no conflicts of interest regarding the publication of this paper.

\section{References}

[1] Kuo, C.L., Liao, W.H. and Shiao, A.S. (2015) A Review of Current Progress in Acquired Cholesteatoma Management. European Archives of Oto-Rhino-Laryngology, 272, 3601-3609. https://doi.org/10.1007/s00405-014-3291-0

[2] Presutti, L., Gioacchini, F.M., Alicandri-Ciufelli, M., Villari, D. and Marchioni, D. (2014) Results of Endoscopic Middle Ear Surgery for Cholesteatoma Treatment: A Systematic Review. ACTA Otorhinolaryngologica Italica, 34, 153-157. http://www.pubmedcentral.nih.gov/articlerender.fcgi?artid=4035841\&tool=pmcent rez\&rendertype $=$ abstract

[3] Kozin, E.D., Gulati, S., Kaplan, A.B., Lehmann, A.E., Remenschneider, A.K., Landegger, L.D., et al. (2015) Systematic Review of Outcomes Following Observational and Operative Endoscopic Middle Ear Surgery. Laryngoscope, 125, 1205-1214. https://doi.org/10.1002/lary.25048

[4] Ayache, S., Tramier, B. and Strunski, V. (2008) Otoendoscopy in Cholesteatoma Surgery of the Middle Ear: What Benefits Can Be Expected? Otology \& Neurotology, 29, 1085-1090. https://doi.org/10.1097/MAO.0b013e318188e8d7 http://content.wkhealth.com/linkback/openurl?sid=WKPTLP:landingpage\&an=001 29492-200812000-00007

[5] Migirov, L., Shapira, Y., Horowitz, Z. and Wolf, M. (2011) Exclusive Endoscopic Ear Surgery for Acquired Cholesteatoma: Preliminary Results. Otology \& Neurotology, 32, 433-436. https://doi.org/10.1097/MAO.0b013e3182096b39

[6] Badr-el-Dine, M. (2002) Value of Ear Endoscopy in Cholesteatoma Surgery. Otology \& Neurotology, 23, 631-635. https://doi.org/10.1097/00129492-200209000-00004

[7] Ito, T., Kubota, T., Watanabe, T., Futai, K., Furukawa, T. and Kakehata, S. (2015) Transcanal Endoscopic Ear Surgery for Pediatric Population with a Narrow External Auditory Canal. International Journal of Pediatric Otorhinolaryngology, 79, 2265-2269. https://doi.org/10.1016/j.ijporl.2015.10.019 
[8] Marchioni, D., Soloperto, D., Rubini, A., Villari, D., Genovese, E., Artioli, F., et al. (2015) Endoscopic Exclusive Transcanal Approach to the Tympanic Cavity Cholesteatoma in Pediatric Patients: Our Experience. International Journal of Pediatric Otorhinolaryngology, 79, 316-322. https://doi.org/10.1016/j.ijporl.2014.12.008

[9] Cohen, M.S., Basonbul, R.A., Kozin, E.D. and Lee, D.J. (2017) Residual Cholesteatoma during Second-Look Procedures Following Primary Pediatric Endoscopic Ear Surgery. Otolaryngology_Head and Neck Surgery, 157, 1034-1040. https://doi.org/10.1177/0194599817729136

[10] Tarabichi, M. (2004) Endoscopic Management of Limited Attic Cholesteatoma. Laryngoscope, 114, 1157-1162. https://doi.org/10.1097/00005537-200407000-00005

[11] Alicandri-Ciufelli, M., Marchioni, D., Kakehata, S., Presutti, L. and Villari, D. (2016) Endoscopic Management of Attic Cholesteatoma: Long-Term Results. Otolaryngologic Clinics of North America, 49, 1265-1270. https://doi.org/10.1016/j.otc.2016.05.015

[12] Presutti, L., Marchioni, D., Mattioli, F., Villari, D. and Alicandri-Ciufelli, M. (2008) Endoscopic Management of Acquired Cholesteatoma: Our Experience. Journal of Otolaryngology—Head \& Neck Surgery, 37, 481-487.

[13] Marchioni, D., Villari, D., Mattioli, F., Alicandri-Ciufelli, M., Piccinini, A. and Presutti, L. (2013) Endoscopic Management of Attic Cholesteatoma: A Single-Institution Experience. Otolaryngologic Clinics of North America, 46, 201-209. https://doi.org/10.1016/j.otc.2012.10.004

[14] Prasad, S.C., Giannuzzi, A., Nahleh, E.A., Donato, G.D., Russo, A. and Sanna, M. (2016) Is Endoscopic Ear Surgery an Alternative to the Modified Bondy Technique for Limited Epitympanic Cholesteatoma? European Archives of Oto-Rhino-Laryngology, 273, 2533-2540. https://doi.org/10.1007/s00405-015-3883-3

[15] Tarabichi, M. (2010) Endoscopic Transcanal Middle Ear Surgery. Indian Journal of Otolaryngology and Head and Neck Surgery, 62, 6-24. https://doi.org/10.1007/s12070-010-0007-7

[16] Glikson, E., Yousovich, R., Mansour, J., Wolf, M., Migirov, L. and Shapira, Y. (2017) Transcanal Endoscopic Ear Surgery for Middle Ear Cholesteatoma. Otology \& Neurotology, 38, e41-e45. https://doi.org/10.1097/MAO.0000000000001395

[17] Sergi, B., Galli, J., Battista, M., De Corso, E. and Paludetti, G. (2014) Dealing with Paediatric Cholesteatoma: How We Changed Our Management. ACTA Otorhinolaryngologica Italica, 34, 138-143.

http://www.pubmedcentral.nih.gov/articlerender.fcgi?artid=4025179\&tool=pmcent rez\&rendertype $=$ abstract

[18] Nogueira, J.F. and Cohen, M.S. (2017) Combined Endoscopic and Microscopic Approaches to Cholesteatoma. Operative Techniques in Otolaryngology-Head and Neck Surgery, 28, 36-38. https://doi.org/10.1016/j.otot.2017.01.006 